\title{
Upregulation of long-noncoding RNA PTPRG-AS1 can predict the poor prognosis and promote migration and invasion in patients with osteosarcoma
}

\author{
RILE GE, PENG YANG and BINGTAO WEN \\ Department of Orthopedics, Peking University International Hospital, Haidian, Beijing 102206, P.R. China
}

Received March 26, 2020; Accepted November 27, 2020

DOI: $10.3892 / 01.2021 .12725$

\begin{abstract}
The present study aimed to determine the expression of the long non-coding RNA PTPRG-AS1 in patients with osteosarcoma, and to explore its role on the prognosis of patients and the process of osteosarcoma cell metastasis. Reverse transcription quantitative-PCR was performed to detect PTPRG-AS1 expression in osteosarcoma tumor tissues and cells (U2OS, SJSA1 and Saos-2), and normal tissues and cells (hFOB1.19). In addition, qPCR and western blotting were also used to detect mRNA and protein expression, respectively, whereas fluorescence in situ hybridization was used to locate the position of PTPRG-AS1 in osteosarcoma cells. Transwell assay was used to determine the migratory and invasive abilities of osteosarcoma cells. The results demonstrated that PTPRG-AS1 was highly expressed in osteosarcoma cells and tissues, which was compared with normal bone cells and adjacent healthy tissues. Furthermore, PTPRG-AS1 expression level in patients with osteosarcoma and lymph node metastasis or distal metastasis was elevated compared with normal tissues. In addition, the results from univariate and multivariate analyses demonstrated that PTPRG-AS1 expression level was significantly associated with Tumor-Node-Metastasis stage $(\mathrm{P}=0.025)$, lymph node metastasis $(\mathrm{P}=0.035)$ and distant metastasis $(\mathrm{P}=0.016)$ in patients with osteosarcoma. PTPRG-AS1 expression level (odd ratio, 3.012; 95\% confidence interval, 1.564-4.219) was also considered as an independent risk factor affecting the 5-year survival rate of patients with osteosarcoma. Furthermore, the 5-year overall survival rate of patients with elevated PTPRG-AS1 expression level $(56.36 \%)$ was significantly lower compared with patients with low PTPRG-AS1 expression level (78.43\%). In addition, PTPRG-AS1 knockdown using small interfering RNA significantly decreased the invasive and migratory abilities of
\end{abstract}

Correspondence to: Professor Wen Bingtao, Department of Orthopedics, Peking University International Hospital, 1 Shengmingyuan Road, Zhongguancun Life Science Park, Beijing 102206, P.R. China

E-mail: bengtaowen@126.com

Key words: osteosarcoma, PTPRG-AS1, metastasis, prognosis osteosarcoma cells in vitro. In summary, PTPRG-AS1 high expression in patients with osteosarcoma may predict the poor prognosis of patients, as PTPRG-AS may have a promoting effect on osteosarcoma cell metastasis.

\section{Introduction}

Osteosarcoma is the most common malignant bone tumor occuring in adolescents or children under the age of 20 years, accounting for $\sim 5 \%$ of the total number of pediatric tumors $(1,2)$. Osteosarcoma can occur in all parts of the bone tissue, although it is more common in the distal femur, proximal tibia and proximal humerus and metaphysis (2). At present, the cause and underlying mechanism of osteosarcoma remain unclear. Neoadjuvant chemotherapy, surgery and radiation therapy are common treatments for patients with osteosarcoma, which allow patients to reach a 5-year overall survival rate of $65-70 \%(3,4)$. However, $25-30 \%$ of patients with osteosarcoma present with metastases, including lung and bone metastases, which significantly decrease their 5-year survival rate (4). Studying the pathogenesis of osteosarcoma would therefore help the development of potential novel targets and new treatment strategies.

Long non-coding RNA (lncRNAs) are defined as transcripts of $>200$ nucleotides in length that are not translated into proteins because they do not have a development reading frame. The Human Genome Project considers lncRNAs as 'garbage sequences' that have accumulated during human evolution. However, during recent years, increasing evidence has demonstrated that lncRNAs are dysregulated in numerous diseases, especially in cancers, and that they serve crucial role in the regulation of various pathophysiological processes, including cell proliferation, apoptosis, necrosis, and autophagy (5-7). The lncRNA PTPRG-AS1 has not only been found to be overexpressed in tumor tissues, including breast cancer (8) and ovarian epithelial cancer (9), but has also been demonstrated to be involved in the regulation of radiosensitivity, metastasis and proliferation of tumor cells $(10,11)$. However, the expression of PTPRG-AS1 and its role in osteosarcoma remain unclear. The present study aimed to determine PTPRG-AS1 expression in patients with osteosarcoma, and to investigate the association between PTPRG-AS1 expression and the prognosis of patients with osteosarcoma. In addition, the function of PTPRG-AS1 on the metastasis of osteosarcoma cells was also assessed. 


\section{Materials and methods}

Patients and tissues. Between January 2014 and January 2019, 106 pairs of osteosarcoma tumor tissue and adjacent healthy tissue were collected from patients at the Peking University International Hospital. The clinicopathological characteristics, including sex, age, anatomical site, histological grade, histologic subtype and clinical stage were collected for each patient with osteosarcoma. The histological subtype and clinical stage of patients with osteosarcoma were divided as before $(12,13)$. The age of the 106 patients ranged from 5 to 32 years (mean age, 11 years). All other clinicopathological characteristics are presented in Table I. All tissues were collected before chemotherapy, radiation or other treatment procedures were conducted, and we followed-up on each patient for up to 5 years after tissue collection. All patients or their guardians provided written informed consent, and the study protocol was approved by the Peking University International Hospital Ethics Committee (Beijing, China; approval no. 2018-042 (BMR). Fresh tissues were stored in liquid nitrogen until subsequent use to detect PTPRG-AS1 expression.

Reverse transcription quantitative (RT-q)PCR. A Cell/tissue Total RNA Isolation kit (cat. no. RC101-01; Vazyme Biotech Co., Ltd.) was used to extract total RNA from tissues and cells. A $20 \mu \mathrm{l}$ of RT-qPCR system was prepared as described in the qPCR master mix kit instructions (cat. no. A6001; Promega Corporation). The following thermocycling conditions were used for qPCR: $95^{\circ} \mathrm{C}$ for $2 \mathrm{~min}$, followed by 40 cycles of $95^{\circ} \mathrm{C}$ for $5 \mathrm{sec}$ and $60^{\circ} \mathrm{C}$ for $30 \mathrm{sec}$, and a final extension at $72^{\circ} \mathrm{C}$ for $5 \mathrm{~min}$. The relative expression of the gene was calculated using the $2^{-\Delta \Delta C q}$ method (14), and $\beta$-actin was used as a loading control. The sequences of the primers were as follows: PTPRG-AS1-2, forward, 5'-CCCTTGAGTGGTCCTCTG TTC-3' and reverse, 5'-GAGCCGGATTTGTCCCAACT-3'; and $\beta$-actin, forward, 5'-AGCCCATCCTTCGAGTACAAA-3' and reverse, 5'-TCT TGGTGCGATAACTGGTGG-3'.

Cell culture and transfection. hFOB1.19, U2OS, SJSA1 and Saos-2 cell lines were purchased from the American Type Culture Collection, cultured in DMEM medium (cat. no. 11965092; Thermo Fisher Scientific, Inc.) supplemented with 10\% FBS (cat. no. 16140071; Thermo Fisher Scientific, Inc.), and placed at $37^{\circ} \mathrm{C}$ in a humidified incubator containing $5 \% \mathrm{CO}_{2}$. Cells $\left(2.5 \times 10^{6}\right)$ were transfected with $50 \mathrm{nmol} / 1$ small interfering (si)RNA using Lipofectamine 2000 reagent (cat. no. 11668019; Thermo Fisher Scientific, Inc.) at $37^{\circ} \mathrm{C}$ for $6 \mathrm{~h}$, according to the manufacturer's instructions. The sequences of the siRNAs used were as follows: si-negative control (NC), forward, 5'-AGGUAG GCCCCUAUCAGCCGGC-3' and reverse, 5'-AUGUGUCGAA UUCGCGUACG-3'; si-PTPRG-AS1-1, forward, 5'-UUCAAA UAUAUUUACUGAGCA-3' and reverse, 5'-CUCAGUAAAU AUAUUUGAAUG-3'; and si-PTPRG-AS1-2, forward, 5'-AUUAUGAUGAAUGUUAACGGG-3' and reverse, 5-CGU UAACAUUCAUCAUAAUUU-3'. After $72 \mathrm{~h}$ of transfections, cells were collected for subsequent experiments.

Transwell assay. Transwell chambers (cat. no. 140629; Thermo Fisher Scientific, Inc.) were used to evaluate the migratory and invasive abilities of cancer cells following to the manufacturer's instructions. Briefly, $0.5 \times 10^{6}$ cells $/ \mathrm{ml}$ in DMEM medium were added into the upper chamber of a 24-well Transwell chamber. The upper chamber was pre-coated with Extra Matrigel (cat. no. BD354248; Becton-Dickinson and Company) to evaluate the invasive ability of cancer cells. Culture medium containing $20 \%$ FBS was added into the lower Transwell chamber. Cells were placed at $37^{\circ} \mathrm{C}$ in a humidified incubator containing $5 \% \mathrm{CO}_{2}$ for $24 \mathrm{~h}$. Subsequently, medium was removed from lower chamber, cells were washed twice with PBS and membrane was fixed with methanol for $5 \mathrm{~min}$ at room temperature and allowed to dry for $30 \mathrm{~min}$. Once membrane was dried, it was stained with crystal violet for $20 \mathrm{~min}$ at room temperature and cells were imaged using a light microscope (x200). ImageJ software [v1.8.0; National Institutes of Health (NIH)] was used to quantify the gray value of spots.

Fluorescence in situ hybridization (FISH). FISH was performed as previously described (15). Briefly, after cells $(50,000$ cells in $200 \mu 1$ DMEM medium added 10\% FBS) or tissues were fixed with 4\% paraformaldehyde (cat. no. LGLS0005; Loogene) for $10 \mathrm{~min}$ at room temperature, they were incubated with a fluorescent probe (Genomeditech Co., Ltd.) that can bind to the human version of the PTPRG-AS1 gene at $37^{\circ} \mathrm{C}$ for $16 \mathrm{~h}$. For cells, the nuclei were counterstained with $5 \mu \mathrm{g} / \mathrm{ml}$ DAPI for $5 \mathrm{~min}$ at room temperature. All samples were visualized using confocal microscopy (LAS AF Lite 4.0, Leica; magnification, x800).

Western blotting. A Whole Cell Extraction Kit (cat. no. OP-0003; Epigentek Group, Inc.) was used to extract total protein from the osteosarcoma cells. Protein concentration was determined using a BCA Protein Quantification Kit (cat. no. E112-01; Vazyme Biotech Co., Ltd.). Subsequently, $45 \mu \mathrm{g}$ proteins were separated by $10 \%$ SDS-PAGE and were transferred onto PVDF membranes. Membranes were blocked using 5\% skimmed milk at room temperature for $1 \mathrm{~h}$ and incubated with the primary antibodies (diluted with $5 \%$ skimmed milk) against E-cadherin (cat. no. ab15148; 1:1,000; Abcam), $\mathrm{N}$-cadherin (cat. no. ab18203; 1:1,000; Abcam), and matrix metalloproteinase-9 (MMP-9) antibody (cat. no. ab38898; 1:2,000; Abcam) and $\beta$-actin (cat. no. sc-69879; 1:3,000; Santacruze) overnight at $4^{\circ} \mathrm{C}$ Membranes were then incubated with goat anti-mouse IgG H\&L (HRP) (cat. no. ab6789; 1:2,000; Abcam) or goat anti-rabbit IgG H\&L (HRP) (cat. no. ab6721; 1:2,000; Abcam) at room temperature for $2 \mathrm{~h}$. Enhanced chemiluminescence reagent (cat. no. WBKLS0100; Beijing Xinjingke Biotechnologies Co., Ltd.) was used to detect the signal on the membrane. ImageJ software (v1.8.0; $\mathrm{NIH}$ ) was used to quantify the gray value of protein bands.

Statistical analysis. SPSS 20.0 software (IBM Corp.) was used to analyze the data. Student $t$-test and $\chi^{2}$ test were used to compare differences between two groups, and one-way ANOVA followed by Tukey's post hoc test was used to compare differences between multiple groups. The multivariate analysis model was used to analyze the association between marker expression levels and clinicopathological characteristics of patients. Log-rank (Mantel-Cox) test was used to compare the survival of patients with high and low levels of PTPRG-AS1 expression. $\mathrm{P}<0.05$ was considered to indicate a statistically significant difference. 


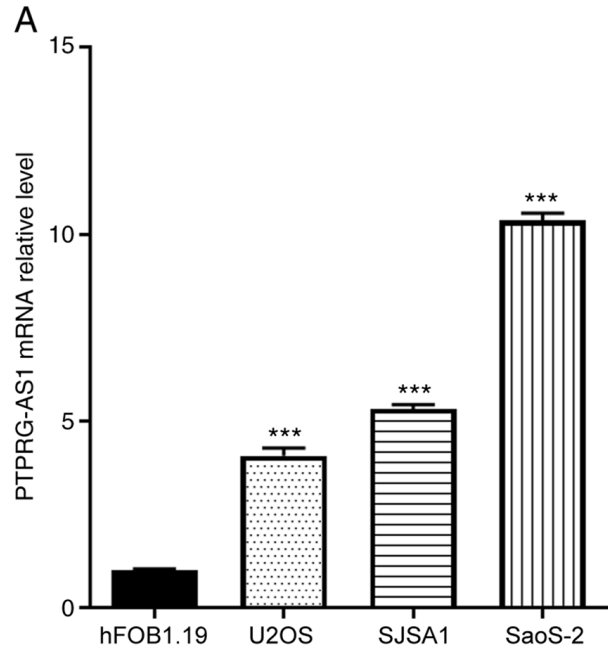

C

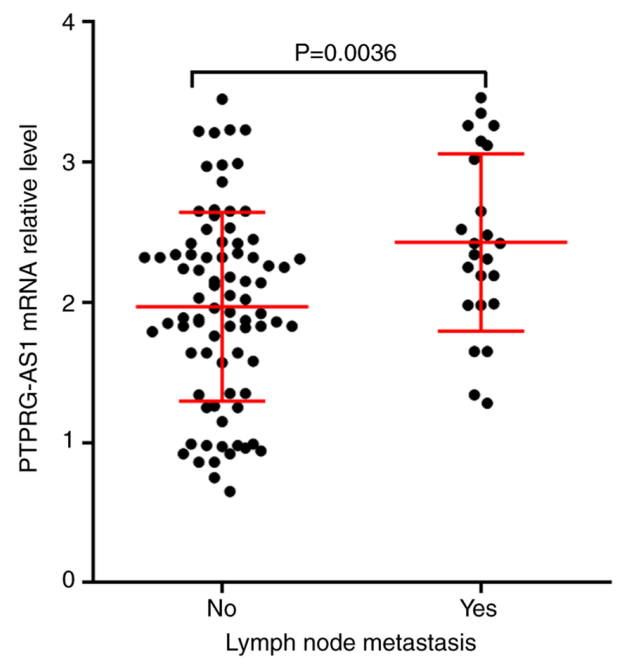

B

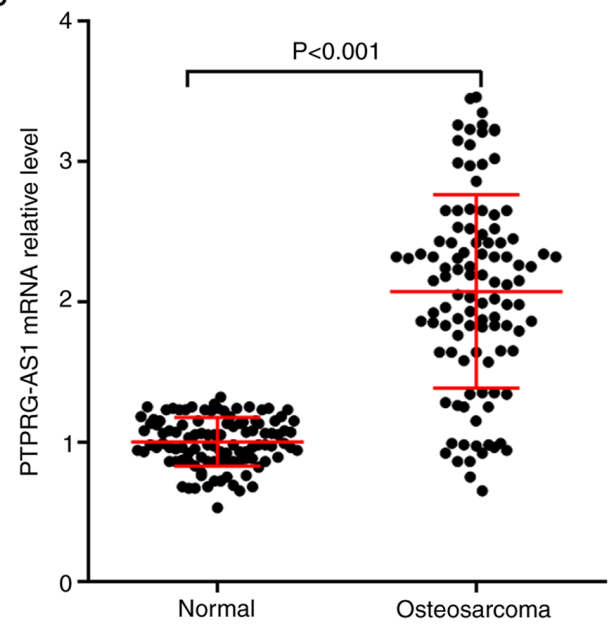

D

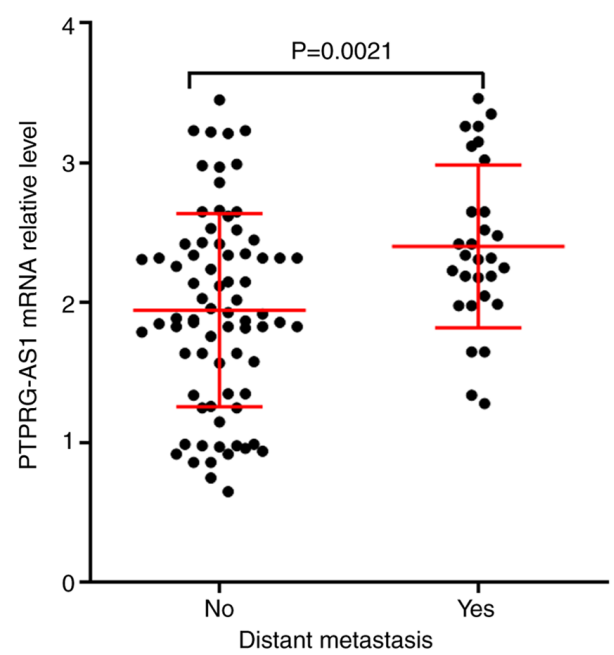

Figure 1. PTPRG-AS1 expression in osteosarcoma cells and tissues. (A) RT-qPCR analysis of PTPRG-AS1 expression in normal human osteoblast (hFOB1.19) and human osteosarcoma cells (U2OS, SJSA1 and Saos2). (B) PTPRG-AS1 expression in human osteosarcoma tissues and paired normal adjacent tissues determined by RT-qPCR. (C and D) Comparison of PTPRG-AS1 expression in osteosarcoma tissues in the absence or presence of (C) lymph node metastasis or (D) distant metastasis. ${ }^{* * * *} \mathrm{P}<0.001$ vs. hFOB1.19 group. RT-qPCR, reverse transcription quantitative PCR.

\section{Results}

PTPRG-AS1 is highly expressed in osteosarcoma cells and tissues. As presented in Fig. 1A, the expression of PTPRG-AS1 was detected in normal human osteoblast (hFOB1.19) and human osteosarcoma cells (U2OS, SJSA1 and Saos2). The results demonstrated that PTPRG-AS1 expression level in human osteosarcoma cells was significantly higher compared with normal human osteoblasts. Furthermore, 106 pairs of human osteosarcoma and normal adjacent tissues were collected and used to detect PTPRG-AS1 expression levels using RT-qPCR. The results demonstrated that PTPRG-AS1 expression level in osteosarcoma tissues was significantly higher compared with normal adjacent tissues (Fig. 1B). In addition, according to the presence of lymph node metastasis or distant metastasis, the 106 patients with osteosarcoma were divided into two groups (presence or absence). The results demonstrated that PTPRG-AS1 expression in patients with lymph node metastasis $(n=24)$ was significantly higher than in patients without lymph node metastasis ( $n=82$; Fig. 1C). In addition, compared with patients without distant metastasis $(\mathrm{n}=77)$, PTPRG-AS1 expression in patients with distant metastasis ( $n=29)$ was significantly higher (Fig. 1D).

Association between PTPRG-AS1 expression and the clinicopathological characteristics of patients with osteosarcoma. According to the expression of PTPRG-AS1 in osteosarcoma tissues, the 106 patients were divided into two groups, the low PTPRG-AS1 expression group $(n=51$; PTPRG-AS1 expression < mean of PTPRG-AS1 expression in the 106 patients) and the high PTPRG-AS1 expression group $(n=55$; PTPRG-AS1 expression $\geq$ mean of PTPRG-AS1 expression in the 106 patients). The association between PTPRG-AS1 expression and the clinicopathological characteristics of patients, including sex, age, anatomical site and histological grade, was subsequently evaluated. As presented in Table I, the expression of PTPRG-AS1 was significantly associated with Tumor-Node-Metastasis (TNM) stage $(\mathrm{P}=0.025)$, lymph node metastasis $(\mathrm{P}=0.035)$ and distant metastasis $(\mathrm{P}=0.016)$ in patients with osteosarcoma, but was not significantly 
Table I. PTPRG-AS1 expression and clinicopathological characteristics of patients with osteosarcoma.

\begin{tabular}{|c|c|c|c|c|c|}
\hline \multirow[b]{2}{*}{ Variable } & \multicolumn{4}{|c|}{ PTPRG expression } & \multirow[b]{2}{*}{ P-value } \\
\hline & $\begin{array}{c}\text { Case } \\
\text { number }\end{array}$ & $\begin{array}{l}\text { Low } \\
(\mathrm{n}=51)\end{array}$ & $\begin{array}{l}\text { High } \\
(n=55)\end{array}$ & $\chi^{2}$ & \\
\hline \multicolumn{6}{|l|}{ Sex } \\
\hline Female & 50 & 26 & 24 & 0.573 & 0.449 \\
\hline Male & 56 & 25 & 31 & & \\
\hline \multicolumn{6}{|l|}{ Age, years } \\
\hline$<14$ & 45 & 23 & 22 & 0.044 & 0.834 \\
\hline$\geq 14$ & 61 & 28 & 33 & & \\
\hline \multicolumn{6}{|l|}{ Anatomical site } \\
\hline Femur & 50 & 20 & 30 & 3.611 & 0.461 \\
\hline Tibia & 21 & 13 & 8 & & \\
\hline Humerus & 15 & 7 & 8 & & \\
\hline Pelvis & 12 & 6 & 6 & & \\
\hline Other & 8 & 5 & 3 & & \\
\hline \multicolumn{6}{|c|}{ Histological grade } \\
\hline G1 & 23 & 11 & 12 & 0.368 & 0.832 \\
\hline G2 & 38 & 15 & 13 & & \\
\hline G3 & 45 & 25 & 20 & & \\
\hline \multicolumn{6}{|c|}{ Histologic subtype } \\
\hline Osteoblastic & 54 & 20 & 25 & 4.066 & 0.397 \\
\hline Chondroblastic & 15 & 10 & 5 & & \\
\hline Fibroblastic & 13 & 9 & 4 & & \\
\hline Telangiectatic & 13 & 6 & 7 & & \\
\hline Other & 11 & 6 & 5 & & \\
\hline \multicolumn{6}{|l|}{ TNM stage } \\
\hline I & 23 & 7 & 16 & 9.357 & 0.025 \\
\hline II & 20 & 7 & 13 & & \\
\hline III & 34 & 17 & 17 & & \\
\hline IV & 29 & 20 & 9 & & \\
\hline \multicolumn{6}{|l|}{$\begin{array}{l}\text { Lymph node } \\
\text { metastasis }\end{array}$} \\
\hline Yes & 24 & 7 & 17 & 4.461 & 0.035 \\
\hline No & 82 & 44 & 38 & & \\
\hline \multicolumn{6}{|l|}{ Distant metastasis } \\
\hline Yes & 29 & 10 & 19 & 5.799 & 0.016 \\
\hline No & 77 & 41 & 26 & & \\
\hline
\end{tabular}

TNM, Tumor-Node-Metastasis.

associated with sex $(\mathrm{P}=0.449)$, age $(\mathrm{P}=0.834)$, anatomical site $(\mathrm{P}=0.461)$, histological grade $(\mathrm{P}=0.832)$ and histological subtype $(\mathrm{P}=0.397)$.

PTPRG-AS1 can predict the poor prognosis of patient with osteosarcoma. The 106 patients were followed up at least once every four months or when the patients came to the hospital for review five years after surgery. The 5-year overall survival of patients was recorded, and the factors influencing the survival of the patients were analyzed using univariate and multivariate

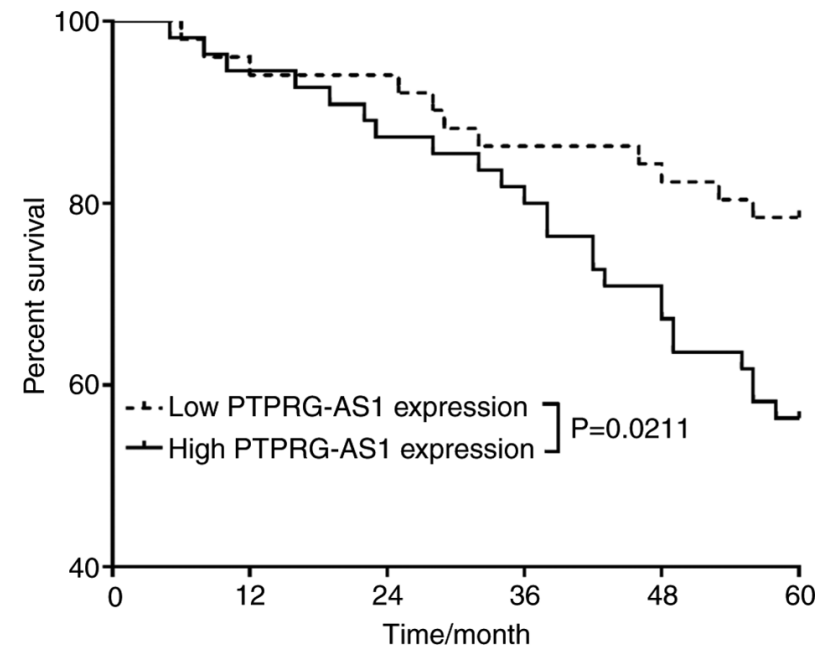

Figure 2. Comparison of the 5-year survival of patients with osteosarcoma according to PTPRG-AS1 expression (high or low).

analyses. As presented in Table II, histological grade [odd ratio $(\mathrm{OR})=1.659$; 95\% confidence interval (CI, 1.844-2.064], TNM stage (OR=1.353; 95\% CI, 1.232-2.564), lymph node metastasis (OR=0.985; 95\% CI, 0.421-1.654), distant metastasis (OR=3.127; 95\% CI, 1.846-4.325) and PTPRG-AS1 expression level (OR=3.012; 95\% CI, 1.564-4.219) were identified as independent risk factors that could affect the 5-year survival of patients with osteosarcoma. Further analysis of the effect of PTPRG-AS1 expression level on the 5-year overall survival rate of patients with osteosarcoma demonstrated that $78.43 \%$ (40/51) of patients with low PTPRG-AS1 expression were still alive 5 years after surgery, whereas only $56.36 \%$ (31/55) of patients with high PTPRG-AS1 expression were still alive 5 years after surgery. The difference between the 5-year survival of patients with osteosarcoma and the difference in PTPRG-AS1 expression was significant ( $\mathrm{P}=0.0211$; Fig. 2).

PTPRG-ASI promotes the migratory and invasive abilities of osteosarcoma cells. The metastasis of cancer cells includes migration and invasion (16), and we used different Transwell chamber to assess invasion and migration in osteosarcoma cells. PPRRG-AS1 expression was knocked down in osteosarcoma cells (Saos-2 and SJSA1) using si-PPRRG-AS1-1 and -2 and the efficiency of the transfection was verified by RT-qPCR and FISH staining. As presented in Fig. 3A, the results from RT-qPCR demonstrated that si-PPRRG-AS1-1 and si-PPRRG-AS1-2 significantly decreased PPRRG-AS1 expression up to 60 and $52 \%$, respectively, in osteosarcoma cells. Therefore, si-PPRRG-AS1-1 and si-PPRRG-AS1-2 were used to knockdown PPRRG-AS1 expression in subsequent experiments. To investigate whether PTPRG-AS1 could regulate the migratory and invasive abilities of osteosarcoma cells, Transwell assays were performed using Saos-2 cells transfected or not with si-PPRRG-AS1-1 and si-PPRRG-AS1-2. The results demonstrated that PTPRG-AS knockdown could significantly decrease the migratory and invasive abilities of osteosarcoma cells (Fig. 3B). Furthermore, PTPRG-AS knockdown could significantly increase the expression of E-cadherin and decrease the expressions of $\mathrm{N}$-cadherin and 
Table II. Analysis of the influencing factors on the survival of patients with osteosarcoma.

\begin{tabular}{|c|c|c|c|c|c|c|}
\hline & \multicolumn{3}{|c|}{ Univariate analysis } & \multicolumn{3}{|c|}{ Multivariate analysis } \\
\hline & $95 \% \mathrm{CI}$ & OR & P-value & $95 \% \mathrm{CI}$ & OR & P-value \\
\hline Sex & $1.567-4.215$ & 2.423 & 0.382 & - & - & - \\
\hline Age & $0.567-3.342$ & 0.984 & 0.412 & - & - & - \\
\hline Anatomical site & $3.129-4.031$ & 3.672 & 0.058 & - & - & - \\
\hline Histological grade & $0.876-2.324$ & 1.561 & 0.042 & $1.844-2.064$ & 1.659 & $<0.001$ \\
\hline Histological subtype & $1.524-3.659$ & 2.227 & 0.067 & - & - & - \\
\hline TNM stage & $0.912-3.021$ & 1.025 & 0.042 & $1.232-2.564$ & 1.353 & 0.049 \\
\hline Lymph node metastasis & $1.324-2.845$ & 1.622 & 0.024 & $0.421-1.654$ & 0.985 & 0.019 \\
\hline Distant metastasis & $1.652-5.126$ & 3.241 & $<0.001$ & $1.846-4.325$ & 3.127 & $<0.001$ \\
\hline PTPRG-AS1 levels & $1.226-5.324$ & 2.984 & 0.016 & $1.564-4.219$ & 3.012 & 0.011 \\
\hline
\end{tabular}

OR, odd ratio; CI, confidence interval.
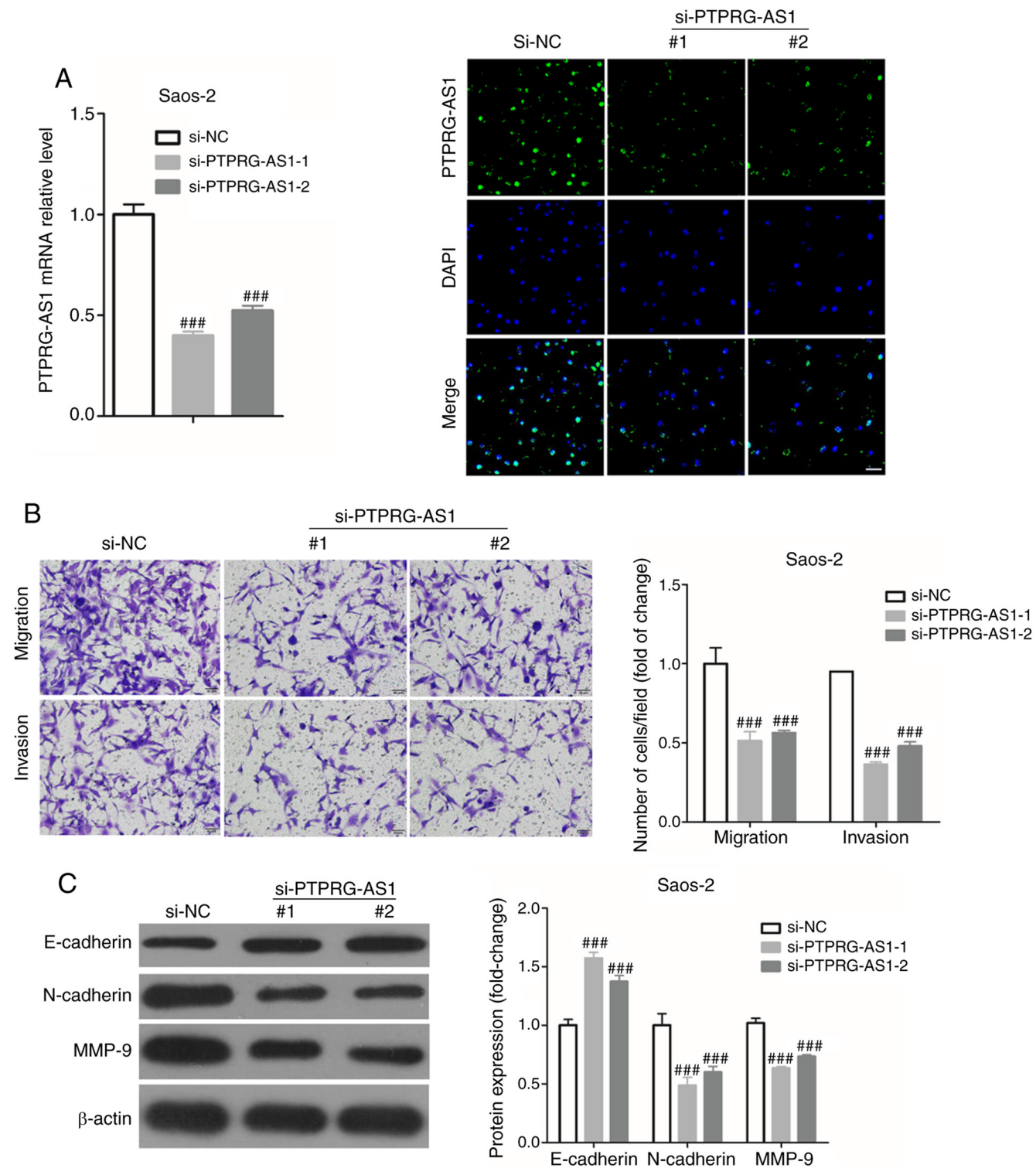

Figure 3. Role of PTPRG-AS1 on the migratory and invasive abilities of Saos-2 cells. (A) Reverse transcription quantitative PCR (left panel) and fluorescence in situ hybridization staining (right panel) confirming PTPRG-AS1 silencing using siRNAs. (B) Transwell assay was used to determine the migratory and invasive abilities of osteosarcoma cells in vitro. (C) Expression of E-cadherin, N-cadherin and MMP-9 in osteosarcoma cells using western blotting.

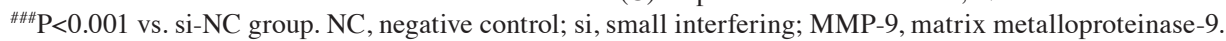



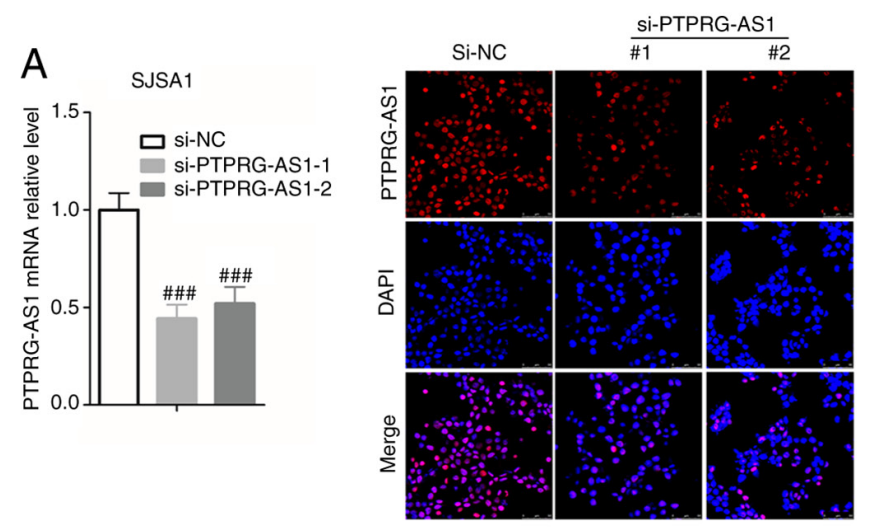

B
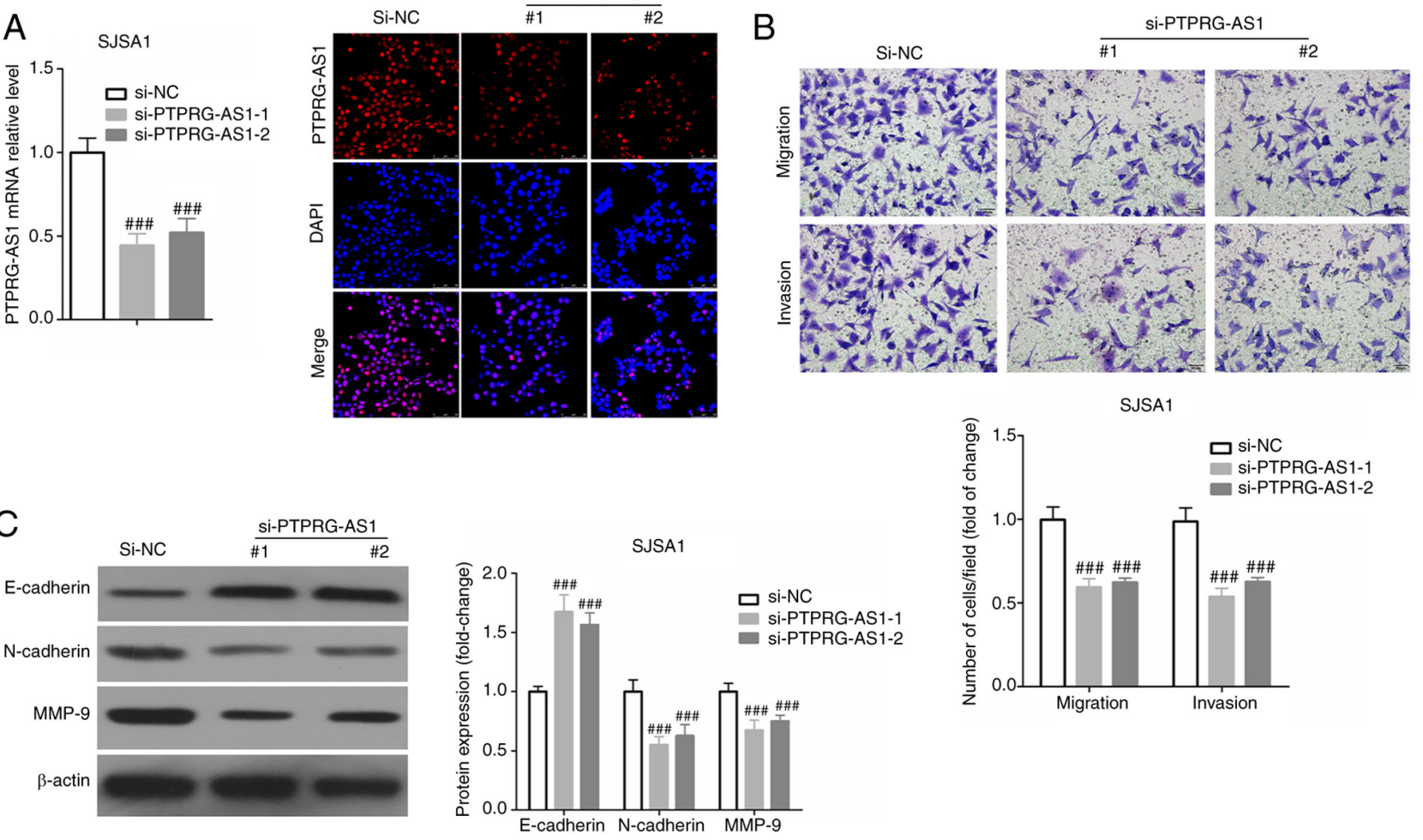

Figure 4. Role of PTPRG-AS1 on the migratory and invasive abilities of SJSA1 cells. (A) Reverse transcription quantitative PCR (left panel) and fluorescence in situ hybridization staining (right panel) confirming PTPRG-AS1 silencing using siRNAs. (B) Transwell assay was used to determine the migratory and invasive abilities of osteosarcoma cells in vitro. (C) Expression of E-cadherin, N-cadherin and MMP-9 in osteosarcoma cells using western blotting.

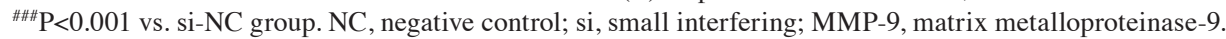

MMP-9 in Saos-2 cells (Fig. 3C). Similar results were obtained following SJSA1 cell transfection with si-PPRRG-AS1-1 and si-PPRRG-AS1-2 (Fig. 4).

\section{Discussion}

Metastasis is one of the main characteristics of cancer cells and cancer-associated mortality often results from metastases rather than from primary tumor growth $(17,18)$. Osteosarcoma, the most common type of primary bone tumor in children, is highly metastatic, and $\sim 25-30 \%$ of patients with osteosarcoma develop metastases (4). Understanding the underlying mechanism of osteosarcoma metastasis to tissues and organs is essential to identify novel therapeutic targets (19). An increasing number of studies have reported that lncRNAs serve crucial roles in the regulation of osteosarcoma cell metastasis. For example, the IncRNA HULC is highly expressed in osteosarcoma cells and tissues and can promote cell metastasis in osteosarcoma (20). Furthermore, Zhao et al (21) reported that the IncRNA HNF1A-AS1 could promote osteosarcoma cell metastasis by activating the $\mathrm{Wnt} / \beta$-catenin signaling pathway. Zhou et al (22) demonstrated that the IncRNA SNHG12 promotes the tumorigenesis and metastasis of osteosarcoma through the upregulation of Notch2 by sponging miR-195-5p. Similarly, GAS5 (23), ZFAS1 (24) and MALAT1 (25) have also been demonstrated to be involved in the regulation of osteosarcoma cell metastasis.

The present study demonstrated that PTPRG-AS1 expression was elevated in osteosarcoma cells and tissues, and that PTPRG-AS1 expression was higher in patients with lymph node metastasis or distal metastasis. Importantly, PTPRG-AS1 expression level in osteosarcoma tissues was not only significantly associated with lymph node metastasis or distal metastasis, but was also significantly associated with the 5 -year survival of patients with osteosarcoma. These results suggested that PTPRG-AS1 may act as an oncogene in osteosarcoma and may therefore be associated with osteosarcoma cell metastasis. PTPRG-AS1 is located on human chromosome 3p14.2 and has been found to play an important role in the regulation of tumor cell behavior. Yi et al (11) reported that PTPRG-AS1 expression is significantly elevated in nasopharyngeal carcinoma tissues using gene sequencing technology, and that PTPRG-AS1 can promote the expression of protein regulator of cytokinesis 1 (PRC1) by binding to miR-194-3p, while PRC1 could enhance the resistance of nasopharyngeal carcinoma cells to radiotherapy and promote the migration of nasopharyngeal carcinoma cells. These results indicated that PTPRG-AS1 serves an indirect role as an oncogene through PRC1. In addition, Xu et al (10) demonstrated that PTPRG-AS1 can regulate the growth of glioma cells by sponging miR-185-5 in vitro.

To evaluate the effect of PTPRG-AS1 on osteosarcoma cell metastasis, the expression of PTPRG-AS1 in osteosarcoma cells was knocked down in the present study, and the changes in the migratory and invasive abilities of cells were determined. The results demonstrated that PTPRG-AS1 silencing could significantly decrease the invasive and migratory abilities of osteosarcoma cells in vitro. PTPRG-AS1 knockdown also significantly increased the expression of E-cadherin and decreased the expression of $\mathrm{N}$-cadherin 
and MMP-9 in osteosarcoma cells. Tumor metastasis is an extremely complicated multi-step process, which includes tumor cell detachment from the primary focal site, invasion of surrounding tissue, entry in the circulatory system, escape from immune surveillance, attachment to distant luminal beds, extravasation into target organ tissue and formation of several secondary tumors $(26,27)$. Cell adhesion plays therefore a crucial role in the invasion and metastasis of cancer cells. Firstly, the separation of cancer cells from the primary cancer is associated with the reduction of homogeneous adhesion of cancer cells, and changes in the adhesion to the stroma is also an important factor that favors tumor metastasis $(26,27)$. Secondly, the formation of homogeneous or heterogeneous tumor plugs in the lumen also results from adhesion $(26,27)$. In addition, during tumor cell migration out of the lumen, adhesion to the lumen endothelium and underlying basement membrane is also an important step during the metastatic process $(26,27)$.

E-cadherin,N-cadherin and MMP-9 are important proteins involved in epithelial-mesenchymal transition (EMT), and EMT is defined as the transformation of epithelial cells to mesenchymal cells $(28,29)$. EMT can alter cell adhesion and enhance cell ability to metastasize and invade, by inducing stem cell characteristics, decreasing apoptosis and aging, and promoting immunosuppression, which not only play a key role in the developmental process, but also participate in tissue healing, organ fibrosis and cancer development (30-32). E-cadherin and $\mathrm{N}$-cadherin are both subtypes of cadherin, which is a calcium-dependent transmembrane glycoprotein that mainly mediates homogeneous adhesion between cells (33,34). A hydrophobic gene in E-cadherin is located in the transmembrane region and an amino terminus is located outside the cell membrane, while the hydroxyl end is located in the cytoplasm and is connected to actin (33). Overall, E-cadherin plays an important role in maintaining cell morphology and regulating cell adhesion (33). However, $\mathrm{N}$-cadherin acts as a promoter to initiate tumor invasion in most malignant tumors, because $\mathrm{N}$-cadherin can decrease cell adhesion by changing cell morphology, and ultimately promotes EMT $(34,35)$. One of the hallmarks of EMT is the loss of epithelial cell integrity, which is accompanied by a decrease in adhesion connections between epithelial cells. The proteolytic digestive function of MMPs is one of the driving factors that cause a decrease in the adhesion connections between epithelial cells $(36,37)$.

In summary, the results from the present study demonstrated that PTPRG-AS1 upregulation in osteosarcoma may promote osteosarcoma cell metastasis, resulting ultimately in the poor prognosis of patients with osteosarcoma. However, the underlying mechanisms by which PTPRG-AS1 may promote osteosarcoma cell metastasis remain unclear and require further investigation.

\section{Acknowledgements}

Not applicable.

\section{Funding}

No funding was received.

\section{Availability of data and materials}

All data generated or analyzed during the present study are available from the corresponding author upon reasonable request.

\section{Authors' contributions}

BW was responsible for the conception and design of the study. RG and PY performed the experiments and analyzed the data. RG and BW confirmed the authenticity of all the raw data. All authors have read and approved the final version of the manuscript.

\section{Ethics approval and consent to participate}

The present study was approved by the Peking University International Hospital Ethics Committee (Beijing, China; approval no. 2018-042 (BMR), and written informed consent was provided by all patients prior to the study start.

\section{Patient consent for publication}

Not applicable.

\section{Competing interests}

The authors declare that they have no competing interests.

\section{References}

1. Ottaviani G and Jaffe N: The epidemiology of osteosarcoma. In: Pediatric and Adolescent Osteosarcoma. Springer, pp3-13, 2009.

2. Lindsey BA, Markel JE and Kleinerman ES: Osteosarcoma Overview. Rheumatol Ther 4: 25-43, 2017.

3. Harrison DJ, Geller DS, Gill JD, Lewis VO and Gorlick R: Current and future therapeutic approaches for osteosarcoma. Expert Rev Anticancer Ther 18: 39-50, 2018.

4. Anderson ME: Update on survival in osteosarcoma. Orthop Clin North Am 47: 283-292, 2016.

5. Wang DQ, Fu P, Yao C, Zhu LS, Hou TY, Chen JG, Lu Y, Liu D and Zhu LQ: Long non-coding RNAs, novel culprits, or bodyguards in neurodegenerative diseases. Mol Ther Nucleic Acids 10: 269-276, 2018.

6. Niu ZS, Niu XJ and Wang WH: Long non-coding RNAs in hepatocellular carcinoma: Potential roles and clinical implications. World J Gastroenterol 23: 5860-5874, 2017.

7. Tehrani SS, Karimian A, Parsian H, Majidinia M and Yousefi B: Multiple functions of long non-coding RNAs in oxidative stress, DNA damage response and cancer progression. J Cell Biochem 119: 223-236, 2018.

8. Iranpour M, Soudyab M, Geranpayeh L, Mirfakhraie R, Azargashb E, Movafagh A and Ghafouri-Fard S: Expression analysis of four long noncoding RNAs in breast cancer. Tumour Biol 37: 2933-2940, 2016.

9. Yang X, Yan M, Chengjiang W, et al: Analysis of LncRNAs and mRNAs expression profiles in ovarian epithelial cancer cell lines by gene microarray. Chinese Journal of Clinical Laboratory Science 36: 384-387,400, 2018.

10. Xu C, Li Z, He T, Yuan B and Ding B: Long noncoding RNA PTPRG-AS1 regulates growth of glioma cells by sponging miR-185-5p. RSC Advances 9: 10870-10880, 2019.

11. Yi L, Ouyang L, Wang S, Li SS and Yang XM: Long noncoding RNA PTPRG-AS1 acts as a microRNA-194-3p sponge to regulate radiosensitivity and metastasis of nasopharyngeal carcinoma cells via PRC1. J Cell Physiol 234: 19088-19102, 2019.

12. Cates JM: Comparison of the AJCC, MSTS, and modified spanier systems for clinical and pathologic staging of osteosarcoma. Am J Surg Pathol 41: 405-413, 2017. 
13. Laurini JA and Cooper K: Pathologic staging of bone and soft tissue tumors: What is new in the eighth edition of the American Joint Committee on Cancer Staging Manual? AJSP 23: 149-156, 2018.

14. Livak KJ and Schmittgen TD: Analysis of relative gene expression data using real-time quantitative PCR and the $2(-\Delta \Delta \mathrm{C}(\mathrm{T}))$ method. Methods 25: 402-408, 2001.

15. Chen ZZ, Huang L, Wu YH, Zhai WJ, Zhu PP and Gao YF: LncSox 4 promotes the self-renewal of liver tumour-initiating cells through Stat3-mediated Sox4 expression. Nat Commun 7: 12598,2016

16. Zhuang J, Huang Y, Zheng W, Yang S, Zhu G, Wang J, Lin X and Ye J: TMEM100 expression suppresses metastasis and enhances sensitivity to chemotherapy in gastric cancer. Biol Chem 401: 285-296, 2020

17. Mumprecht V and Detmar M: Lymphangiogenesis and cancer metastasis. J Cell Mol Med 13: 1405-1416, 2009.

18. Su Z, Yang Z, Xu Y, Chen Y and Yu Q: Apoptosis, autophagy, necroptosis, and cancer metastasis. Mol Cancer 14: 48, 2015.

19. Yang J and Zhang W: New molecular insights into osteosarcoma targeted therapy. Curr Opin Oncol 25: 398-406, 2013.

20. Sun XH, Yang LB, Geng XL, Wang R and Zhang ZC: Increased expression of lncRNA HULC indicates a poor prognosis and promotes cell metastasis in osteosarcoma. Int J Clin Exp Pathol 8: 2994-3000, 2015.

21. Zhao H,Hou W, Tao J,Zhao Y, Wan G, Ma C and Xu H: Upregulation of lncRNA HNF1A-AS1 promotes cell proliferation and metastasis in osteosarcoma through activation of the Wnt/3-catenin signaling pathway. Am J Transl Res 8: 3503-3512, 2016.

22. Zhou S, Yu L, Xiong M and Dai G: LncRNA SNHG12 promotes tumorigenesis and metastasis in osteosarcoma by upregulating Notch2 by sponging miR-195-5p. Biochem Biophys Res Commun 495: 1822-1832, 2018.

23. Wang Y and Kong D: LncRNA GAS5 represses osteosarcoma cells growth and metastasis via sponging MiR-203a. Cell Physiol Biochem 45: 844-855, 2018.

24. Liu G, Wang L, Han H, Li Y, Lu S, Li T and Cheng C: LncRNA ZFAS1 promotes growth and metastasis by regulating BMI1 and ZEB2 in osteosarcoma. Am J Cancer Res 7: 1450-1462, 2017.

25. Dong Y, Liang G, Yuan B, Yang C, Gao R and Zhou X: MALAT1 promotes the proliferation and metastasis of osteosarcoma cells by activating the PI3K/Akt pathway. Tumour Biol 36: 1477-1486, 2015
26. Valastyan S and Weinberg RA: Tumor metastasis: Molecular insights and evolving paradigms. Cell 147: 275-292, 2011.

27. Steeg PS: Tumor metastasis: Mechanistic insights and clinical challenges. Nat Med 12: 895-904, 2006.

28. Lamouille S, Xu J and Derynck R: Molecular mechanisms of epithelial-mesenchymal transition. Nat Rev Mol Cell Biol 15: 178-196, 2014.

29. Yang J and Weinberg RA: Epithelial-mesenchymal transition: At the crossroads of development and tumor metastasis. Dev Cell 14: 818-829, 2008.

30. Radisky DC: Epithelial-mesenchymal transition. J Cell Sci 118: 4325-4326, 2005

31. Kalluri R and Weinberg RA: The basics of epithelial-mesenchymal transition. J Clin Invest 119: 1420-1428, 2009.

32. Gonzalez DM and Medici D: Signaling mechanisms of the epithelial-mesenchymal transition. Sci Signal 7: re8-re8, 2014.

33. Korpal M,Lee ES, Hu G and Kang Y: The miR-200 family inhibits epithelial-mesenchymal transition and cancer cell migration by direct targeting of E-cadherin transcriptional repressors ZEB1 and ZEB2. J Biol Chem 283: 14910-14914, 2008.

34. Nakajima S, Doi R, Toyoda E, Tsuji S, Wada M, Koizumi M, Tulachan SS, Ito D, Kami K, Mori T, et al: N-cadherin expression and epithelial-mesenchymal transition in pancreatic carcinoma. Clin Cancer Res 10: 4125-4133, 2004.

35. Nguyen PT, Kudo Y, Yoshida M, Kamata N, Ogawa I and Takata T: $\mathrm{N}$-cadherin expression is involved in malignant behavior of head and neck cancer in relation to epithelial-mesenchymal transition. Histol Histopathol 26: 147-156, 2011.

36. Radisky ES and Radisky DC: Matrix metalloproteinase-induced epithelial-mesenchymal transition in breast cancer. J Mammary Gland Biol Neoplasia 15: 201-212, 2010.

37. Malemud CJ: Matrix metalloproteinases (MMPs) in health and disease: An overview. Front Biosci 11: 1696-1701, 2006.

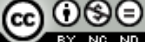

This work is licensed under a Creative Commons Attribution-NonCommercial-NoDerivatives 4.0 International (CC BY-NC-ND 4.0) License. 